\title{
Nefrectomia videolaparoscópica em Graxaim-do-campo (Pseudalopex gymnocercus) com displasia renal ${ }^{1}$
}

\author{
João P.S. Feranti², Adriana C. da Motta ${ }^{3}$, Marília T. Oliveira², Fernando Wiecheteck², \\ Luís F.D. Corrêa ${ }^{2}$, Jefferson S. Pires ${ }^{4}$, Carlos A.C. Beck ${ }^{5}$ e Maurício V. Brun ${ }^{2 *}$
}

\begin{abstract}
Feranti J.P.S., Motta A.C., Oliveira M.T., Wiecheteck F., Corrêa L.F.D., Pires J.S., Beck C.A.C. \& Brun M.V. 2014. [Laparoscopic nephrectomy in Pampas fox (Pseudalopex gymnocercus) with renal dysplasia.] Nefrectomia videolaparoscópica em Graxaim-do-campo (Pseudalopex gymnocercus) com displasia renal. Pesquisa Veterinária Brasileira 34(8):776-779. Departamento de Clínica de Pequenos Animais, Centro de Ciências Rurais, Universidade Federal de Santa Maria, Avenida Roraima 1000, Camobi, Santa Maria, RS 97105-900, Brazil.E-mail: mauriciovelosobrun@hotmail.com

Pampas fox (Pseudalopex gymnocercus) inhabits east-central South America, from southern Brazil to eastern Bolivia. The renal dysplasia comprises a spectrum of abnormalities and is often reported in dogs of various breeds; but this disease reports involve mostly necropsy findings. Congenital renal diseases in dogs have a progressive character, so that treatment is limited and directed to retard the disease. This report describes a laparoscopic nephrectomy in pampas fox with renal dysplasia. Right kidney disease was suspected by ultrasound and intravenous urography. For the surgical procedure we used three portal accesses $(10,10,5 \mathrm{~mm})$, rigid endoscope $10 \mathrm{~mm} / 0^{0}$ and clips for hemostasis of the renal vessels. Due the absence of reports of laparoscopic nephrectomy in pampas fox and scarcity of surgical techniques for this species, the operative technique used was similar to that used in the domestic dog. The pampas fox was monitored for 30 days after surgery without the occurrence of complications. This report demonstrates that the proposed technique is appropriate to perform laparoscopic nephrectomy in pampas fox.
\end{abstract}

INDEX TERMS: Pampas fox, Pseudalopex gymnocercus, renal dysplasia, nephrectomy, surgery, canid, wild, kidney disease, laparoscopy.

RESUMO-- Graxains-do-campo habitam o centro-leste da América do Sul, desde o sudeste do Brasil e leste da Bolívia. A displasia renal compreende um espectro de anomalias e é frequentemente relatada em cães de diversas raças, sendo a maioria dos relatos achados de necropsia. A maior

\footnotetext{
${ }^{1}$ Recebido em 16 de agosto de 2013.

Aceito para publicação em 4 de junho de 2014.

${ }^{2}$ Departamento de Clínica de Pequenos Animais (DCPA), Laboratório de Cirurgia Experimental, Centro de Ciências Rurais (CCR), Universidade Federal de Santa Maria (UFSM), Avenida Roraima 1000, Santa Maria, RS 97105-900, Brasil. Bolsista CNPq/Brasil. *Autor para correspondência: mauriciovelosobrun@hotmail.com

${ }^{3}$ Docente do Curso de Medicina Veterinária da Universidade de Passo Fundo (UPF), Campus I, Cx. Postal 611, Passo Fundo, RS 99001-970, Brasil.

${ }^{4}$ Docente do Curso de Medicina Veterinária da Universidade da Região da Campanha (URCAMP), Praça Getúlio Vargas 47, Centro, Alegrete, RS 97542-570, Brasil.

${ }^{5}$ Docente do Curso de Medicina Veterinária, Universidade Federal do Rio Grande do Sul (UFRGS), Av. Bento Gonçalves 9090, Porto Alegre, RS 91540-000.
}

parte das doenças renais congênitas nos cães apresenta caráter progressivo, portanto o tratamento é limitado e direcionado a fim de retardar a progressão da doença renal estabelecida. No presente relato é descrita a realização de nefrectomia laparoscópica em graxaim-do-campo (Pseudalopex gymnocercus) com displasia renal. Suspeitou-se de doença no rim direito por meio de ultrassonografia e urografia excretora. Para o procedimento cirúrgico foram utilizados três portais $(10,10,5 \mathrm{~mm})$, endoscópio rígido de $10 \mathrm{~mm} / 0^{\circ}$ e clipes para hemostasia dos vasos renais. A técnica cirúrgica utilizada neste canídeo selvagem foi semelhante à usada em cão doméstico, devido à escassez de relatos em cirurgias em graxaim e a inexistência de descrições de nefrectomia videolaparoscópica em graxaim-do-campo. 0 animal foi acompanhado por um período de 30 dias pós-cirurgia sem a ocorrência de complicações. 0 presente relato demonstra que a técnica proposta foi adequada para a realização de nefrectomia videolaparoscópica em Graxaim-do-campo. 
TERMOS DE INDEXAÇÃO: Graxaim-do-campo, Pseudalopex gymnocercus, displasia renal, nefrectomia, cirurgia, canídeo, selvagens, doença renal, laparoscopia.

\section{INTRODUÇÃO}

Graxains-do-campo habitam o centro-leste da América do Sul, desde o sudeste do Brasil e leste da Bolívia, e são fortemente associados com habitats de campos abertos dos pampas, cerrados e chacos (Lucherini et al. 2004). Medindo até um metro de comprimento, com peso médio entre 4,2 e $5,9 \mathrm{~kg}$ (Lucherini et al. 2004), tem seu padrão de atividade diurno e noturno (Di Bitetti et al. 2009).

A nefrectomia laparoscópica foi inicialmente descrita em humanos para a remoção de massa neoplásica em paciente de 85 anos no início da década de 1990 (Clayman et al. 1991). Desde então, o procedimento vem sendo realizado rotineiramente em medicina, tanto para o tratamento de doenças renais como na obtenção de órgãos para transplante (Fabrizio et al. 1999). Já em animais, as primeiras extirpações renais por laparoscopia foram realizadas com o intuito de desenvolver técnica para futura aplicação em humanos (Clayman et al. 1990).

No final do século XX, Brun et al. (2000) foram os primeiros a realizar nefrectomia laparoscópica em cão parasitado por Dioctophyma renale. Durante a cirurgia não ocorreram complicações, e o paciente apresentou excelente recuperação pós-operatória. Posteriormente, utilizando técnica semelhante, Brun et al. (2002) relataram a aplicação com sucesso desse procedimento em outro cão com dioctofimose. Já Beck et al. (2000a) desenvolveram um modelo de obstrução ureteral por laparoscopia para a produção de hidronefrose em cães, onde todos os animais cursaram com a doença, sendo posteriormente tratados com sucesso por meio de nefrectomia laparoscópica (Beck et al. 2000b).

A displasia renal compreende um espectro de anomalias. Em crianças, as malformações do aparelho urinário constituem a doença mais frequentemente diagnosticada na etapa pré-natal. Distinguem-se quatro tipos de displasias, a doença renal policística autossômica recessiva infantil (Potter tipo I), displasia renal multicística (Potter tipo II), doença renal policística autossômica dominante (Potter tipo III) e a displasia renal cística (Potter tipo IV). Frequentemente, é enfermidade relatada em cães de diversas raças, incluindo Shih-Tzu, Lhasa Apso, Golden Retriever, Rodhesian, Bernese, Boxer, Mastiff e Rotweiller (Lobetti et al. 1996, Olenick 1999, Hoppe \& Karlstam 2000, Abraham et al. 2003, Volkweis et al. 2012), porém em animais selvagens ainda a literatura é carente no que se diz respeito a essa doença. De outra forma, a execução de nefrectomia videolaparoscópica em canídeos selvagens ainda é tema praticamente inexplorado.

0 presente relato tem por objetivo descrever o emprego da nefrectomia videolaparoscópica em graxaim-do-campo (Pseudalopex gymnocercus) com displasia renal.

\section{RESULTADOS}

Um graxaim-do-campo (Pseudalopex gymnocercus), do sexo feminino, pesando $6 \mathrm{~kg}$, foi encaminhado para ultrassonografia abdominal em aula prática na disciplina de diag- nóstico por imagem. Durante o exame, não foi possível localizar adequadamente o rim direito, e não foram constatadas alterações nos demais órgãos intracavitários. Devido a suspeita de doença renal foi realizada urografia excretora, que demonstrou que somente o rim esquerdo apresentava excreção do material de contraste. Frente a esses achados, optou-se pela laparoscopia exploratória, devido à possibilidade de associar o diagnóstico definitivo com a terapêutica cirúrgica (Azziz et al. 1989) e pela possibilidade clínica de se tratar de um caso de dioctofimose, já que que havia suspeita ultrassonográfica desta afecção.

Após a contenção química, foi realizada ampla tricotomia abdominal. Em seguida, o paciente foi intubado e mantido em anestesia geral inalatória com isoflurano vaporizado em oxigênio a $100 \%$ em circuito semiaberto, por meio do sistema de Baraka. Realizou-se a monitorização transoperatória dos sinais vitais pela mensuração das frequências cardíaca e respiratória, além da saturação de oxigênio por meio de oximetria de pulso. Após o posicionamento do paciente em decúbito dorsal, introduziu-se a agulha de Verres através da cicatriz umbilical. A cavidade foi insuflada com $\mathrm{CO}_{2}$ até a pressão de $10 \mathrm{mmHg}$ e velocidade de $2 \mathrm{~L} / \mathrm{min}$. Após obtenção de pneumoperitônio, o paciente foi colocado em decúbito lateral esquerdo, com inclinação de aproximadamente $30^{\circ}$ em relação à mesa operatória. Realizou-se incisão na região lateral direita, através da qual foi posicionado trocarte de $11 \mathrm{~mm}$ que serviu de passagem para o endoscópio rígido de $10 \mathrm{~mm} / 0^{0}$. Outros dois trocarteres (11 $\mathrm{mm}$ e $6 \mathrm{~mm}$ ) foram colocados na parede abdominal lateral direita em disposição triangular em relação ao primeiro.

Por meio de manipulação das vísceras abdominais com pinças Kelly e Babcock, foi possível identificar o rim direito (Fig.1A). 0 órgão apresentava diminuição de seu volume, alteração de formato e aderência ao lobo lateral direito do fígado. Frente aos achados macroscópicos e a ausência de excreção de contraste na urografia, optou-se pela nefrectomia total. Com a utilização de tesoura de Metzenbaum, foi realizada a dissecação do hilo renal e o isolamento dos vasos renais (Fig.1C). A artéria e a veia renal foram individualmente ocluídas com dois clipes de titânio e seccionadas na distância média entres os clipes (Fig.1B). Na sequência, realizou-se a dissecação do ureter direito do peritônio e estruturas adjacentes, bem como a sua oclusão e secção próximo à bexiga de forma semelhante à descrita para os vasos. 0 rim foi dissecado do lobo hepático e removido da cavidade em conjunto com um dos trocartes de $11 \mathrm{~mm}$. Após a verificação da ausência de hemorragia, as cânulas foram retiradas, a cavidade foi desinsuflada e as incisões de acesso suturadas em dois planos com náilon monofilamentar.

0 paciente foi acompanhado por um período de 30 dias pós-cirurgia sem a ocorrência de complicações, sendo então encaminhado para o mini zoológico da Universidade de Passo Fundo (UPF). 0 exame microscópico do órgão extirpado demonstrou desorganização do parênquima com atrofia glomerular e espaços glomerulares dilatados contendo material eosinofílico homogêneo associados à atrofia e dilatação tubular cística com cilindros hialinos. Foi observado marcado espessamento da cápsula renal por 


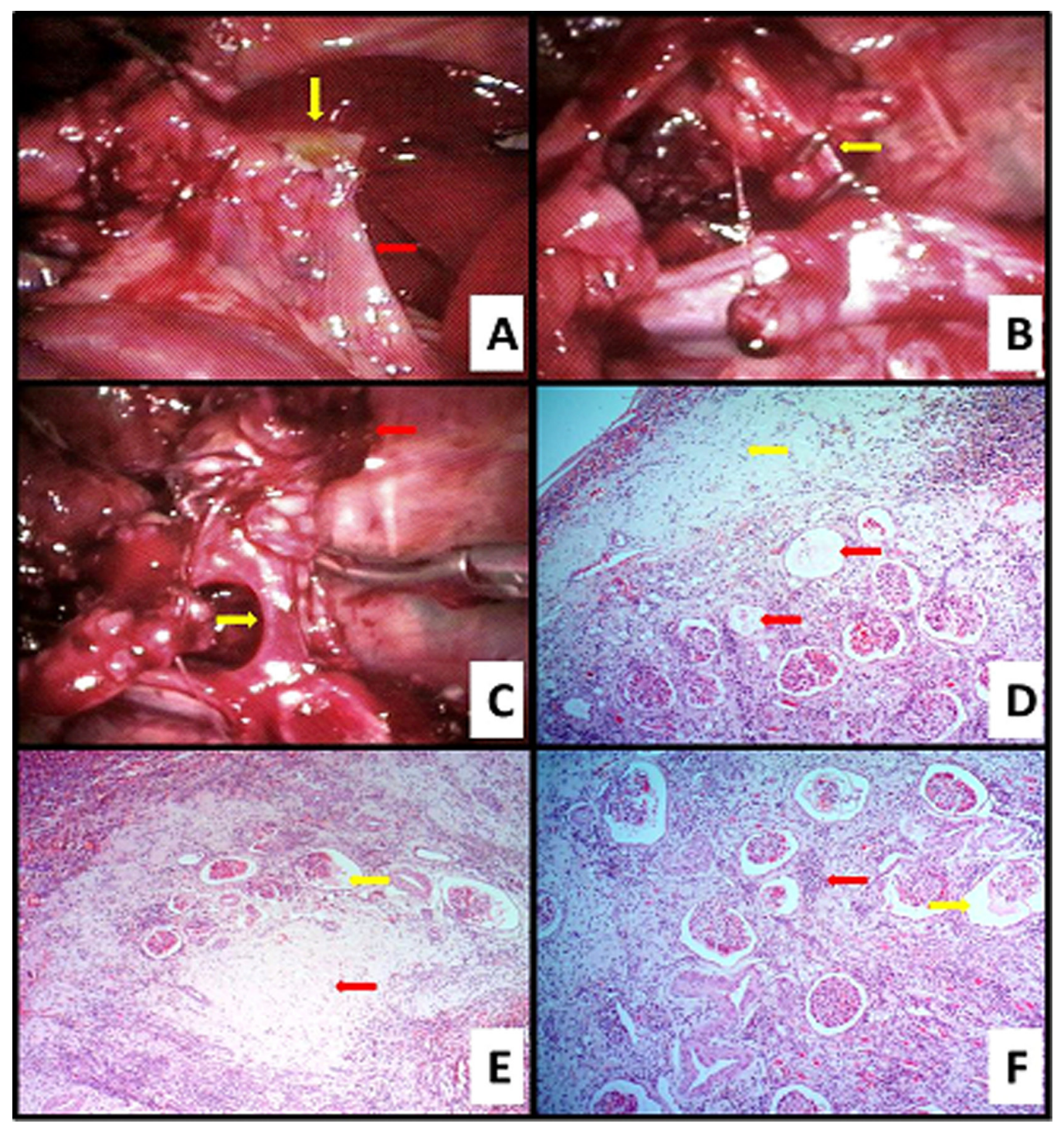

Fig.1. Representação de momentos do procedimento de nefrectomia videolaparoscópica em graxaim-do-campo (A,B,C), bem como do exame microscópico do rim extirpado (D,E,F). (A) Visualização do rim direito, de coloração alterada (seta amarela), aderido em lobo hepático lateral direito e em parte de omento (seta vermelha). (B) Vasos renais dissecados e ocluídos com a aplicação de três clipes de titânio (seta), para posterior secção. (C) Artéria e veia renal dissecada (seta amarela) para posterior hemostasia, note o rim sendo elevado (seta). (D) Rim, displasia renal. Córtex. Atrofia tubular cística, espaços glomerulares dilatados contendo material eosinofílico homogêneo (setas vermelhas). Espessamento da cápsula renal por fibrose (seta). HE, 100x. (E) Rim, displasia renal. Córtex. Fibroplasia intersticial (seta), espaços glomerulares dilatados contendo material eosinofílico (seta amarela). HE, 100x. (F) Rim, displasia renal. Córtex. Atrofia tubular com cilindros hialinos, espaço glomerular dilatado contendo material eosinofílico (seta) e infiltrado inflamatório mononuclear (seta vermelha). HE, 100x.

fibrose, por vezes, associado à hemorragia. Observou-se também, fibroplasia e infiltrado mononuclear intersticial. Na medula, verificou-se atrofia tubular cística com cilindros hialinos, fibroplasia e infiltrado mononuclear intersticial (Fig.1D,E,F). A partir desses achados, foi diagnosticada displasia renal.

\section{DISCUSSÃO}

Diversos são os achados clínicos dos animais com displasia renal como poliúria, polidipsia, perda de peso, caquexia, sinais esses compatíveis com insuficiência renal crônica. Como o paciente do presente caso tratava-se de um animal recolhido pela patrulha ambiental da cidade de Passo Fundo, não possuía histórico clínico. Contudo, por ocasião do recolhimento, o animal apresentava apático com mucosas pálidas. Após o tratamento de suporte e estabilização do quadro clínico, o paciente foi submetido à avaliação ultrassonográfica do abdome sob indução anestésica.

$\mathrm{Na}$ microscopia, podem-se classificar as mudanças morfológicas da displasia renal três grupos (Picutt \& Lewis 1987): I - lesões primárias caracterizadas por glomérulos imaturos ou fetais, tecido mesangial persistente, ducto metanéfrico persistente, epitélio tubular atípico ou metaplasia disontogênica; II - lesões degenerativas ou inflamatórias, representadas por fibrose intersticial, nefrite/pielonefrite túbulo intersticial, mineralização distrófica, atrofia glomerular cística e lipidose glomerular; III - alterações compensatórias com metaplasia ou hipertrofia glomerular 
e tubular. No presente caso, observaram-se alterações morfológicas compatíveis com os primeiros dois grupos.

Para diagnosticar possíveis doenças renais podem ser utilizados diversos artifícios de imagem, como radiografias contrastadas, ultrassonografias, tomografia computadorizada e a ressonância magnética. Como os meios diagnósticos disponíveis (ultrassonografia, radiografia simples e contrastada - urografia excretora) não foram conclusivos para o diagnóstico definitivo da possível doença renal, apenas mostrando que o rim contralateral apresentava-se adequadamente funcional, optou-se pela laparoscopia exploratória, devido à possibilidade de associar o diagnóstico definitivo com a possível terapêutica cirúrgica.

A maior parte das doenças renais congênitas nos cães apresenta caráter progressivo, portanto o tratamento é limitado e direcionado a fim de retardar a progressão da doença renal estabelecida (Polzin et al. 2004). Considerando-se que o tratamento indicado para os casos de displasia renal consiste na extirpação do órgão, optou-se pela realização de nefrectomia videolaparoscópica devido às numerosas vantagens que o acesso tem em relação à cirurgia convencional, conforme tem sido demonstrado em diversos trabalhos tanto em seres humanos com em animais (Savassi-Rocha et al. 1997, Rodrigues et al. 2008). Quanto a esse aspecto, considerou-se principalmente as possíveis vantagens relacionadas à recuperação pós-operatória, fato muito importante ao se trabalhar com animais silvestres. A técnica cirúrgica utilizada neste canídeo selvagem foi semelhante à usada em cão doméstico, devido à escassez de relatos em cirurgias em graxaim e a inexistência de relatos de nefrectomia videolaparoscópica em na referida espécie.

Para obtenção do pneumoperitônio os autores indicam para nefrectomia a técnica aberta com o paciente posicionado em decúbito lateral, tal como descrito por Secchi et al. (2010). Essa indicação baseou-se no fato de a obtenção do pneumoperitônio pela técnica fechada com a agulha de Veress estar associada a vários relatos de lesões viscerais (Yerdel et al. 1999, Zaraca et al. 1999), assim como pelo considerável risco de lesões em consequência da punção às cegas do primeiro trocarte (Malm et al. 2004, Azevedo et al. 2004), principalmente ao se trabalhar com animais de menor porte como no presente caso.

O uso da videolaparoscopia na medicina veterinária têm se desenvolvido cada vez mais, tanto como meio diagnóstico como terapêutico. Porém, em animais selvagens, o uso é mais restrito a poucos centros nacionais. Assim, busca-se aperfeiçoamento de técnicas usadas rotineiramente em seres humanos para o uso em animais selvagens, procurando-se reduzir o tamanho e o número de lesões de acesso, na busca de recuperação pós-operatória precoce e maior controle de dor.

\section{CONCLUSÃO}

0 presente relato demonstra que a técnica proposta é adequada para a realização de nefrectomia videolaparoscópica em graxaim-do-campo.

\section{REFERÊNCIAS}

Abraham L., Beck C. \& Slocombe R.F. 2003. Renal dysplasia and urinary trat infection in a Bull Mastiff Puppy. Aust. Vet. J. 81:336-339.
Azevedo J.L.M.C., Guindalini R.S.C., Azevedo A.C., Paiva V.C., Delorenzo A. \& Moreira M.B. 2004. Avaliação do posicionamento da agulha de Veress durante o estabelecimento do pneumoperitônio pela técnica fechada em porcos. Revta Col. Bras. Cir. 31(5):318-323.

Azziz R., Steinkampf M.P. \& Murphy A. 1989. Postoperative recuperation: relation to the extent of endoscopic surgery. Fertil. Steril. 51:10611064.

Beck C.A.C., Pippi N.L., Brun M.V., Gonçalves G.F., Leme M.C., Oliveira S.T., Stedile R. \& Braga F.V. 2000a. Modelo de hidronefrose unilateral em cães através de obstrução do ureter por abordagem laparoscópica. Ciênc. Anim. Bras. 1:177.

Beck C.A.C., Pippi N.L., Brun M.V., Gonçalves G.F., Leme M.C., Oliveira S.T., Stedile R. \& Braga F.V. 2000b. Nefrectomia laparoscópica em cães com hidronefrose unilateral. Ciênc. Anim. Bras. 1:178.

Brun M.V., Mariano M.B., Beck C.A.C., Antunes R., Genari M. \& Pigatto J.A.T. 2002. Nefrectomia em um canino parasitado por Dioctophyma renale. Arq. Ciênc. Vet. Zool. 5(1):145-152.

Brun M.V., Pippi N.L., Beck C.A.C., Mariano M.B., Pigatto J.A.T., Antunes R., Gaiga L.H. \& Freire C. 2000. Nefrectomia laparoscópica para a terapêutica de dioctofimose em cães. Ciênc. Anim. Bras. 1:175.

Clayman R.V., Kavoussi L.R., Long S.R., Dierks S.M., Meretyk S. \& Soper N.J. 1990. Laparoscopic nephrectomy: initial report of pelviscopic organ ablation in the pig. J. Endourol. 4(3):247-252.

Clayman R.V., Kavoussi L.R., Soper N.J., Dierks S.M., Meretyk S., Darcy M.D., Roemer F.D., Pingleton E.D., Thompson P.G. \& Long S.R. 1991. Laparoscopic nephrectomy: initial case report. J. Urol. 146:278-282.

Di Bitetti M.S., Di Blanco Y.E., Pereira J.A., Paviolo A. \& Perez I.J. 2009. Time partitioning favors the coexistence of sympatric Crab-eating Foxes ( $\mathrm{Cer}$ docyon thous) and Pampas Foxes (Lycalopex gymnocercus). J. Mammal. 90:479-490.

Fabrizio M.D., Ratner L.E., Montgomery R.A. \& Kavoussi L.R. 1999. Laparoscopic live donor nephrectomy. Urol. Clin. North Am. 26(1):247-256.

Lobetti R.G., Pearson J. \& Jimenez M. 1996. Renal dysplasia a Rhodesian ridgeback dog. J. Small Anim. Pract. 37:552-555.

Lucherini M., Pessino M. \& Farias A.A. 2004. Canid Specialist Group. Pampas fox least concern, p.63-68. Disponível em <http://www.canids.org> Acessado em 28 de Março de 2013.

Malm C., Savassi-Rocha P.R., Gheller V.A., Oliveira H.P., Lamounier A.R. \& Foltyneck V. 2004. Ovário-histerectomia: estudo experimental comparativo entre as abordagens laparoscópica e aberta na espécie canina. Intra-operatório I. Arq. Bras. Med. Vet. Zootec. 56(4):457-466.

Olenick C.L. 1999. Congenital renal dysplasia and psychogenic polydipsia in a Bernese Mountain dog. Can. Vet. J. 40:425-426.

Picutt C.A. \& Lewis R.M. 1987. Microscopic features of canine renal dysplasia. Vet. Pathol. 24:156-163.

Polzin D.J., Osborne C.A. \& Bartges J.W. 2004. Insuficiência Renal Crônica, p.1721-1749. In: Ettinger S.J. \& Feldman E.C. (Eds), Tratado de Medicina Interna Veterinária. Guanabara Koogan, Rio de Janeiro.

Rodrigues M.A., Oliveira V.F.F. \& Poveda V.B. 2008. Vantagens e desvantagens da colecistectomia por videolaparoscopia. Janus 5(7):119-128.

Savassi-Rocha P.R., Ferreira J.T. \& Costa Diniz M.A. 1997. Laparoscopic cholecystectomy in Brazil: analysis of 33,563 cases. Int. Surg. 82:208-213.

Secchi P., Valle S.F., Brun M.V., Motta A.C., Rausch S.F., Messina S.A. \& Vieira M.I.B. 2010. Nefrectomia videolaparoscópica para tratamento da dioctofimose em um cão. Acta Scient. Vet. 38(1):85-89.

Volkweis F.S., Almeida A.M.S., Wong L., Mulinari F. \& Santo Júnior H.L. 2012. Displasia renal em cão da raça Rotweiller. Arq. Bras. Med. Vet. Zootec. 64(6):1511-1514.

Yerdel M.A., Karayalcin K., Koyuncu A., Akin B., Koksoy C., Turkcapar AG., Erverdi N., Alacayir I., Bumin C. \& Aras N. 1999. Direct trocar insertion versus Veress needle insertion in laparoscopic cholecystectomy. Am. J. Surg. 177:247-249.

Zaraca F., Catarci M., Gossetti F., Mulieri G. \& Carboni M. 1999. Routine use of open laparoscopy: 1,006 consecutive cases. J. Laparoendosc. Adv. Surg. Tech. 9:75-80. 